\title{
Aortic Rupture due to Co-localization of Aortic Intimal Myofibroblastic Sarcoma and Urothelial Carcinoma: A Unique Case Report
}

\author{
Yutaka Ohashi ${ }^{1}$, Yuki Yamamoto ${ }^{2}$, Akitoshi Yamada ${ }^{3}$, Kazuhiro Yamamoto ${ }^{4}$, Keisuke Arai ${ }^{5}$, \\ Fumiko Namba ${ }^{6}$, Naokazu Miyamoto ${ }^{6}$, Masaru Tomita ${ }^{6}$, Takeshi Fukumoto ${ }^{7}$, \\ Tsutomu Minamikawa ${ }^{1}$ and Masahiro $\mathrm{Oka}^{8}$
}

\begin{abstract}
:
We herein report a unique case of aortic rupture due to co-localization of aortic intimal myofibroblastic sarcoma (IMFS) and urothelial carcinoma (UC). A 76-year-old man who was being followed up after surgery for UC 5 years earlier developed aortic rupture and underwent emergency surgery. Intraoperatively, a tumorous mass on the luminal side of the aortic arch was found near the rupture. A histopathological analysis of the mass revealed aortic IMFS. Furthermore, co-localization of IMFS and UC cells was found near the rupture. The fragility of the aortic wall due to co-localization of IMFS and UC was believed to contribute to the aortic rupture.
\end{abstract}

Key words: aortic arch rupture, aortic sarcoma, co-localization, intimal myofibroblastic sarcoma, urothelial carcinoma

(Intern Med 60: 269-274, 2021)

(DOI: 10.2169/internalmedicine.5191-20)

\section{Introduction}

Aortic sarcomas are extremely rare and aggressive tumors originating from the aorta, with a propensity for arterial embolization, disseminated metastases and rapid clinical deterioration (1). In 1985, Wright et al. (2) categorized aortic sarcomas as intimal and mural types. In 2004, Thalheimer et al. (3) proposed further classifying aortic sarcomas as intimal angiosarcomas originating from endothelial cells of the intima, intimal myofibroblastic sarcomas (IMFSs) originating from mesenchymal cells of the intima, and mural sarcomas originating from myofibroblastic cells of the media or adventitia.

We herein report a case of aortic IMFS that was co- localized with urothelial carcinoma (UC) at the aortic arch, causing aortic arch rupture.

\section{Case Report}

A 76-year-old Japanese man noticed a painful mass on the right palatine tonsil and was referred to the Department of Otolaryngology at Kita-Harima Medical Center from an otolaryngology clinic. The patient had undergone transurethral resection of bladder tumor (TURBT) surgery five years earlier. A pathological evaluation of the TURBT specimens had revealed high-grade invasive UC, at least pT1. After the first TURBT procedure, the UC recurred in the bladder several times and was treated each time by TURBT.

At his current presentation, an otolaryngologic examina-

${ }^{1}$ Department of Dentistry and Oral Surgery, Kita-Harima Medical Center, Japan, ${ }^{2}$ Department of Diagnostic Pathology, Kita-Harima Medical Center, Japan, ${ }^{3}$ Department of Cardiovascular Surgery, Kita-Harima Medical Center, Japan, ${ }^{4}$ Department of Otorhinolaryngology, Kita-Harima Medical Center, Japan, ${ }^{5}$ Department of Gastroenterology, Kita-Harima Medical Center, Japan, ${ }^{6}$ Department of Diagnostic Radiology, KitaHarima Medical Center, Japan, ${ }^{7}$ Division of Dermatology, Department of Internal Related, Kobe University Graduate School of Medicine, Japan and ${ }^{8}$ Department of Dermatology, Kita-Harima Medical Center, Japan

Received: April 28, 2020; Accepted: July 19, 2020; Advance Publication by J-STAGE: September 12, 2020

Correspondence to Dr. Masahiro Oka, masahiro_oka@kitahari-mc.jp 


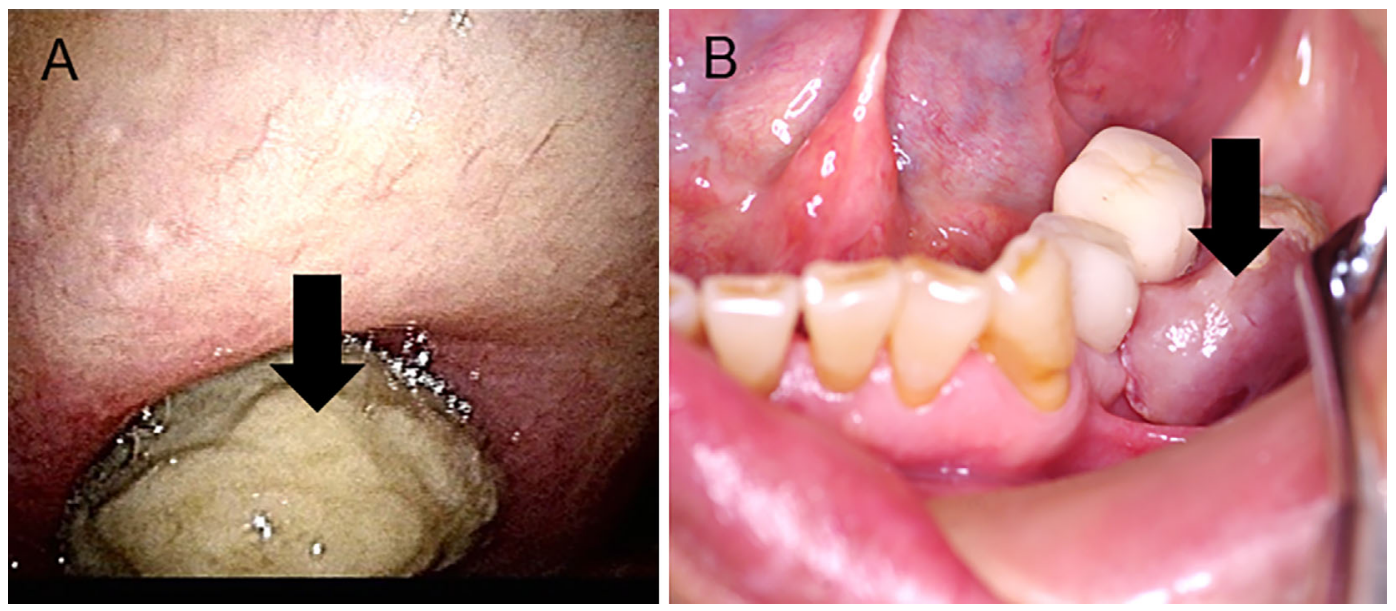

Figure 1. Clinical appearance of the pharyngeal and oral lesions. Yellow-white soft mass on the right palatine tonsil (A: black arrow). Violet-grey mass on the buccal surface of the lower left mandibular gingiva near the molar tooth (B: black arrow).

tion revealed a yellow-white soft mass on the right palatine tonsil (Fig. 1A). Hospitalization and a histopathological examination after en bloc surgical resection of the mass under general anesthesia was scheduled for approximately three weeks later. In the interim, the patient noticed a mass on the lower left mandibular gingiva and was referred to the Department of Dentistry and Oral Surgery at the center from a dental clinic. An oral examination revealed a violet-grey mass measuring $20 \times 10 \times 10 \mathrm{~mm}$ on the buccal surface of the lower left mandibular gingiva near the molar tooth (Fig. 1B). The mass was biopsied. Subsequently, the patient was admitted to the Department of Otolaryngology for the scheduled surgical resection of the right palatine tonsillar mass.

On the evening of the admission day, the patient was found comatose in his bed. Whether or not the patient was in a state of shock was unclear, since records of his vital signs were missing. At that time, his percutaneous arterial oxygen saturation $\left(\mathrm{SpO}_{2}\right)$ was $100 \%$. Intravenous infusion of saline and oxygen inhalation $(10 \mathrm{~L} / \mathrm{min})$ were commenced. Five minutes after commencing saline infusion and oxygen inhalation, his blood pressure and heart rate were 94/34 $\mathrm{mmHg}$ and $100 \mathrm{bpm}$, respectively. At this time, he was able to react to his name being called. His electrocardiogram was normal, while echocardiography showed an echo-free space around the heart. Brain computed tomography (CT) revealed no apparent intracranial lesions. Chest $\mathrm{CT}$ showed pericardial effusion/hematoma (Fig. 2A-C, E) and typical findings of aortic pseudoaneurysm (Fig. 2B). The above findings led us to suspect cardiac tamponade due to aortic rupture. In addition, an oval contrast defect was observed on the luminal side of the aortic arch near the rupture (Fig. 2C, D), suggesting a tumorous mass in the aortic arch. However, we were unable to identify the type or origin of the mass. Chest CT coincidentally showed multiple nodules in the right lung (Fig. 2E) and several enlarged mediastinal lymph nodes (Fig. 2F).
The patient underwent emergency surgery for total arch replacement. When the pericardium was opened, a large volume of bloody pericardial effusion with hematoma (90 g) was observed. During the operation, fragile intimal thickening of the aortic arch was found around the rupture. In addition, a tumorous, white, mutton fat-like translucent mass measuring $15 \times 15 \times 10 \mathrm{~mm}$ (Fig. 3A, B) was found on the luminal side of the aortic arch, at a point a few centimeters distal to the rupture. Aortic dissection was not identified. Furthermore, several enlarged mediastinal lymph nodes were confirmed. The tumorous mass, resected aortic arch $(80 \times 30$ $\mathrm{mm}$ ) (Fig. 3C), and three mediastinal lymph nodes were submitted for a histopathological examination. The patient recovered consciousness at the end of the aortic repair surgery.

A histopathological examination of the tumorous mass revealed proliferation of atypical cells with anisonucleosis in the myxoid tissue (Fig. 4A). Immunohistochemically, these cells were positive for $\alpha$-smooth muscle actin (SMA) (Fig. 4B) and negative for CK AE1/3, CD31, and CD34. Approximately $30 \%$ of the cells were stained by Ki- 67 . Murine double minute (MDM)2 (4) was not detected by dual color in situ hybridization (DISH). Based on these findings, the tumorous mass was diagnosed as aortic IMFS.

A histopathological examination of the aortic arch near the rupture revealed co-localization of two different kinds of tumor cells. First, non-epithelioid atypical cells with anisonucleosis were diffusely distributed over a major part of the surface of the polypoid growth in the aortic intima (Fig. 4C, D). The immunohistochemical findings of these atypical cells showed positivity for $\alpha$-SMA (Fig. 4E, F). These atypical cells were diagnosed as aortic IMFS. In addition to the diffuse distribution of IMFS cells on the surface of the polypoid growth in the aortic intima, a small aggregation of atypical epithelioid cells with anisonucleosis was observed in the deep aortic media in the same section (Fig. 4C, G, H). Immunohistochemically, these cells were 

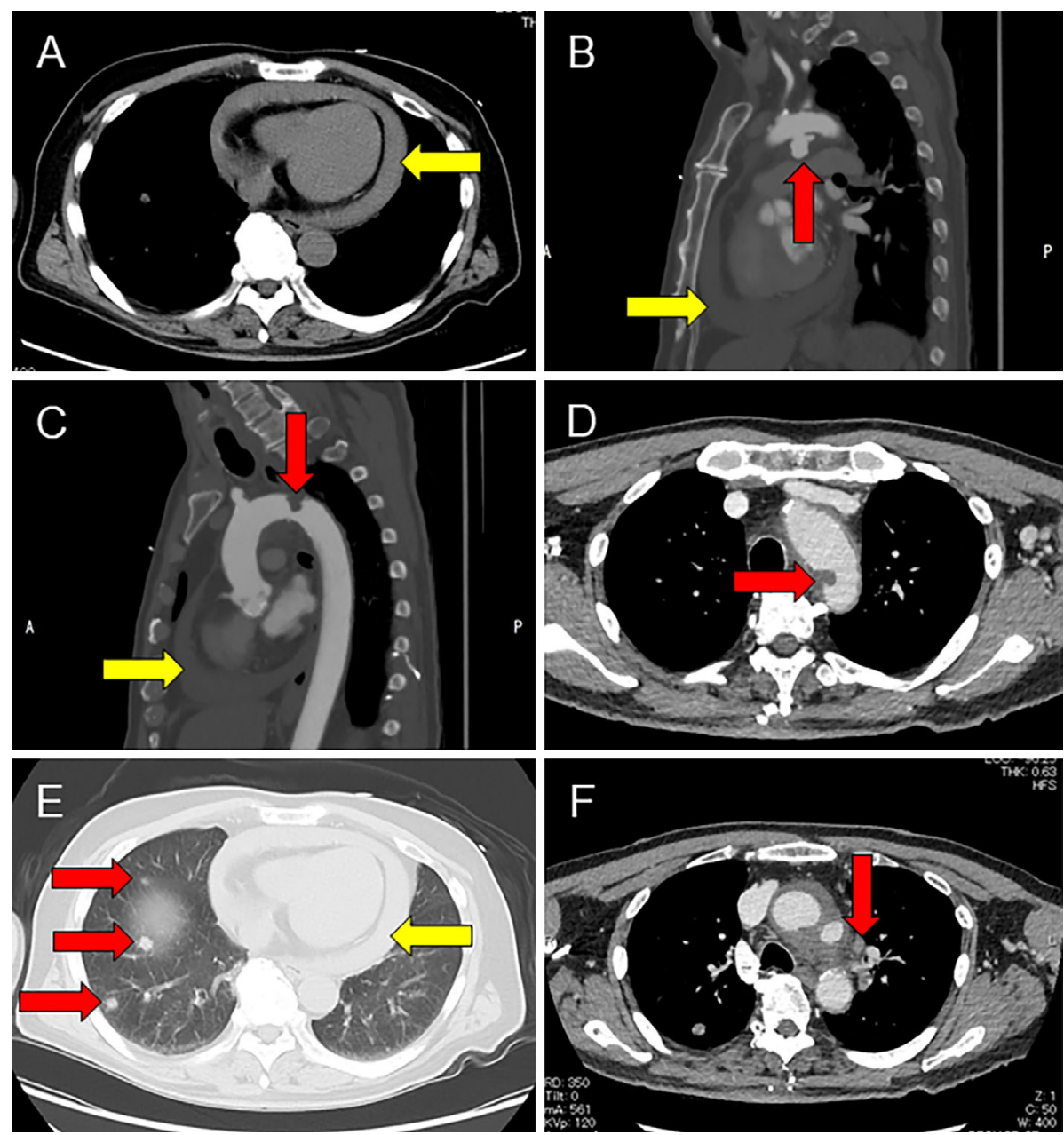

Figure 2. Chest CT. Pericardial effusion/hematoma. A: yellow arrow (axial image); B: yellow arrow (sagittal image); C: yellow arrow (sagittal image); E: yellow arrow (axial image). Ruptured saccular bump at the aortic arch [B: red arrow (sagittal image)]. Oval contrast defect on the luminal side of the aortic arch near the rupture [C: red arrow (sagittal image); D: red arrow (axial image)]. Multiple nodules in the right lung [E: red arrows (axial image)]. Mediastinal lymph node [F: red arrow (axial image)].

negative for $\mathrm{CK}$ AE1/3, CK7, CK20, CD3, CD20, CD56, CD30, D2-40, CD31, S-100, HMB45, and desmin. Approximately $50 \%$ of the cells were stained by Ki-67. These cells were diagnosed as UC cells. A histopathological examination of the three mediastinal lymph nodes revealed that one had diffuse infiltration of atypical cells with anisonucleosis. The immunohistochemical findings of these cells were similar to those of UC cells in the aortic media. Approximately $50 \%$ of the cells were stained by Ki-67. These cells were diagnosed as UC cells.

Postoperatively, the mass on the right palatine tonsil continued to grow, resulting in interference with respiration. On postoperative day 16, his $\mathrm{SpO}_{2}$ abruptly decreased to $80 \%$, and urgent tracheotomy was performed. The tumorous mass $(52 \times 45 \times 32 \mathrm{~mm})$ was resected en bloc two days after the tracheotomy. A histopathological examination revealed that the mass was the palatine tonsil, most of which had been replaced with metastatic cells of aortic IMFS. The mass on the lower left mandibular gingiva was also histopathologically diagnosed as metastasis of aortic IMFS.
On postoperative day 21 , the patient developed ileus and underwent urgent laparotomy two days later. Two tumors were found in the intestine, both of which were resected. A histopathological examination revealed that both tumors were metastatic lesions of aortic IMFS.

The patient was transferred to the palliative care ward to receive best supportive care. He died of respiratory failure, perhaps due to metastatic lesions of aortic IMFS in the proximal part of his trachea, on postoperative day 61. Since an autopsy was not performed, the detailed nature of the multiple nodules in the right lung remained unknown.

\section{Discussion}

Although Thalheimer et al. (3) categorized intimal sarcomas as intimal angiosarcomas and IMFSs, as described above, it seems that the concept of IMFS has not been widely recognized, as no intimal sarcoma case has been reported under the name of IMFS, while a relatively large number of cases of intimal angiosarcoma have been re- 


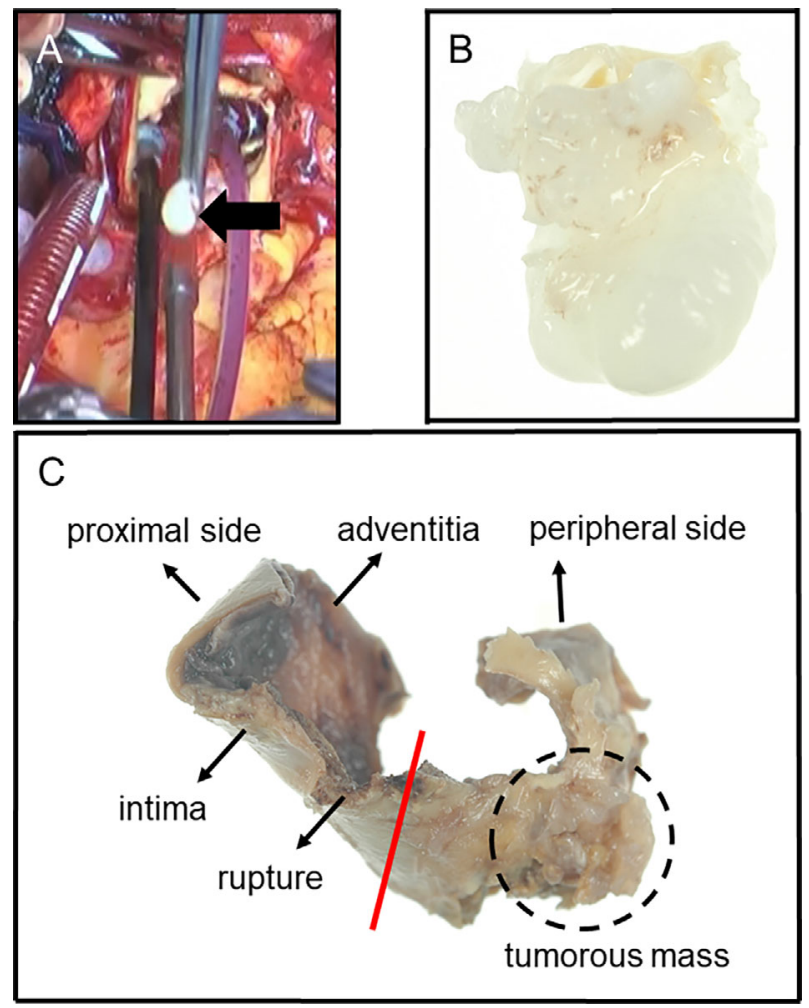

Figure 3. Macroscopic findings. A white, mutton fat-like translucent tumorous mass measuring $15 \times 15 \times 10 \mathrm{~mm}$ was found in the aortic arch during the operation (A: black arrow). The tumorous mass after fixation (B). Resected aortic arch after fixation (C). Note that the proximal half of the resected tissue was inverted by fixation. The red line shows the incision line for making specimens for histopathological analysis.

ported. However, we found at least two reported cases $(5,6)$ that correspond to IMFS in terms of the immunohistochemical findings of positivity for $\alpha$-SMA and negativity for CD31 and CD34, as seen in the present case. These immunohistochemical findings may be able to be used to differentiate IMFS from aortic intimal angiosarcoma.

The infiltration of UC cells in the aorta observed in the present case is very intriguing. Three possible metastatic pathways of UC cells from the primary bladder lesion to the aorta can be considered. The first possibility is that infiltration of UC cells in the aorta occurred by hematogenous metastasis from the primary UC lesion in the bladder. However, to our knowledge, there have been no case reports of hematogenous tumor metastasis to the aorta from the primary tumor. Thus, if this was indeed the route of metastasis in the present case, the phenomenon is of extreme interest. The second possibility is that UC cells from the bladder first metastasized to organs other than the aorta and then subsequently metastasized to the aorta. In the present case, after confirming no lung metastasis of UC cells by chest X-ray at the first TURBT, a further search for UC metastasis using imaging tests was unfortunately not performed. Thus, we do not know if metastasis of UC cells had already occurred in organs other than the aorta before the UC cells reached the aorta. However, the patient had multiple nodules in the right lung on CT when the aortic UC lesion was found. Assuming that these nodules were metastasis of UC cells, a plausible hypothesis is that UC cells from the primary bladder tumor metastasized first to the lung and then secondarily to the aorta. Of course, it is also possible that the pulmonary nodules were metastases of IMFS cells, since these cells easily spread to the lung (1). The third possibility is that the UC lesion in the aorta was a result of direct invasion of tumor cells from mediastinal lymph nodes containing metastasized UC cells. Although extremely rare, tumor infiltration of the aorta caused by direct invasion from tumor-containing tissues around the aorta has been reported for certain kinds of tumors, such as leiomyosarcoma (7) and neurofibroma (8). However, this possibility seems to be very unlikely, since the mediastinal lymph nodes containing metastasized UC cells were not contiguous with the aortic arch, as seen on CT (Fig. 2F) as well as intraoperatively.

Although at least 165 cases of aortic sarcoma have been reported (1), the present case is the first case of aortic sarcoma co-localized with another kind of tumor within the same region of the aorta. The coexistence of IMFS and UC in the present case might have been a coincidence. However, an interesting idea to explain their coexistence is that IMFS itself might play a pathologic role in enhancing the metastasis of other tumors. Specifically, IMFS might have produced certain factors that induce angiogenesis, inflammation, or necrosis, resulting in the enhancement of hematogenous metastasis of UC cells to the site of the pre-existing IMFS. Further investigation is necessary to prove this possibility.

Aortic sarcomas sometimes present as acute aortic rupture or dissection $(5,6,9,10)$. In addition, there have been a few aortic rupture cases due to the invasion of tumors besides aortic sarcomas $(7,8)$. These facts indicate that tumor infiltration of the aorta is a risk factor for aortic rupture. In the present case, the area of distribution of the IMFS in the aorta was much larger than that of UC (Fig. 4C). Thus, it is likely that aortic IMFS cells played a more important role in aortic wall fragility and aortic rupture than UC cells.

Many organs and tissues, including bone, lung, lymph nodes, liver, skin, and bowel, have been reported as metastatic sites of aortic sarcomas (1). However, metastasis to the pharynx and oral cavity, as observed in the present case, has not been reported. Our experience suggests that clinicians should consider the possibility of aortic sarcomas when they come across unusual sarcomatous lesions in the pharynx and oral cavity.

Since intimal aortic sarcomas, including IMFS, are extremely rare, the recognition of these entities and a high level of suspicion are necessary to reach the correct diagnosis. Based on an analysis of 165 cases of aortic sarcomas, Rusthoven et al. (1) described the therapeutic strategy in patients with aortic sarcoma. They recommend noninvasive angiography with CT and magnetic resonance (MR) imaging for the radiologic evaluation of aortic sarcomas, with MR angiography regarded as the gold standard due to superior 

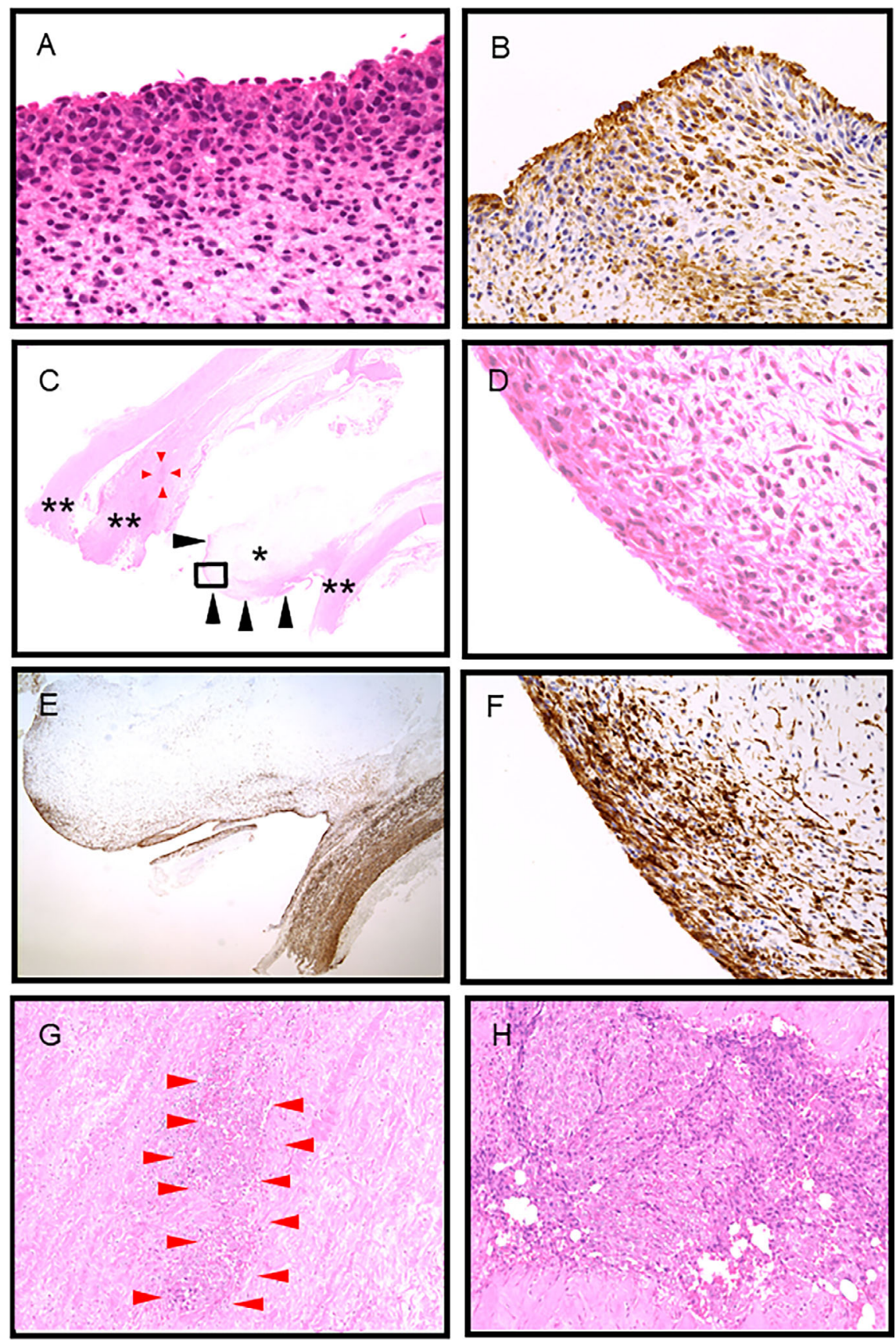

Figure 4. Histopathological and immunohistochemical findings of the tumorous mass in the aorta and aortic arch. The tumorous mass consisted of diffuse proliferation of atypical cells with anisonucleosis in the myxoid tissue [Hematoxylin and Eosin (H\&E) staining, original magnification $\times 200]$ (A). The atypical cells in (A) were positive for $\alpha$-SMA (B). Two different kinds of tumor cells were observed in the same section of the aortic arch near the rupture (H\&E staining, original magnification $\times 4)(C)$. Non-epithelioid atypical cells with anisonucleosis were diffusely distributed on most of the surface of the polypoid growth of the aortic intima (*) (C: black arrowheads). Most of the aortic intima in the polypoid growth was occupied by myxoid tissue. Magnified image of the inset in $\mathrm{C}$ (H\&E staining, original magnification $\times 200$ ) $(D)$. The non-epithelioid atypical cells in Fig. 4C and $D$ were positive for $\alpha$-SMA (E, F, respectively). Aortic media (** in C) was also positive for $\alpha$-SMA (E). In addition to the diffuse distribution of non-epithelioid atypical cells (IMSF cells), a small aggregation of atypical epithelioid cells with anisonucleosis was observed in the deep aortic media (C: red arrowheads). Note that the distribution area of non-epithelioid atypical cells (IMSF) was much larger than that of atypical epithelioid cells (UC cells). Magnified image of the entire aggregation of atypical epithelioid cells in $\mathrm{C}(\mathrm{H} \& \mathrm{E}$ staining, original magnification $\times 200)(\mathrm{G}$ : red arrowheads). UC cells observed in another section (H\&E staining, original magnification $\times 200)(\mathrm{H})$. 
soft tissue characterization and the ability to distinguish an enhancing tumor from the frequently associated thrombus. They concluded that radical surgical resection is the only curative approach for aortic sarcomas. For patients with unresectable disease because of medical and anatomic considerations, debulking surgery and radiotherapy might mitigate local disease progression. They also stated that chemotherapy is associated with a significant improvement in the median survival.

The authors state that they have no Conflict of Interest (COI).

\section{References}

1. Rusthoven CG, Liu AK, Bui MM, et al. Sarcomas of the aorta: a systemic review and pooled analysis of published reports. Ann Vasc Surg 28: 515-525, 2014.

2. Wright EP, Glick AD, Virmani R, Page DL. Aortic intimal sarcoma with embolic metastases. Am J Surg Pathol 9: 890-897, 1985.

3. Thalheimer A, Fein M, Geissinger E, Franke S. Intimal angiosarcoma of the aorta: report of a case and review of the literature. $\mathrm{J}$ Vasc Surg 40: 548-553, 2004.
4. Bode-Lesniewska B, Zhao J, Speel EJ, et al. Gains of 12q13-14 and overexpression of $\mathrm{mdm} 2$ are frequent findings in intimal sarcomas of the pulmonary artery. Virchows Arch 438: 57-65, 2001.

5. Tanaka M, Tabata M, Shimokawa T, Takanashi S. The rupture of descending thoracic aorta due to the necrosis of aortic intimal sarcoma. Interact Cardiovasc Thorac Surg 10: 462-463, 2010.

6. Dedeilias P, Koletsis E, Nenekidis I, et al. Intimal aortic sarcoma mimicking ruptured thoracoabdominal type IV aneurysm. A rare case report and review of the literature. J Cardiothorac Surg 6: 162, 2011.

7. Cantwell CP, Stack J. Abdominal aortic invasion by leiomyosarcoma. Abdom Imaging 31: 120-122, 2006.

8. Falcone JL, Go MR, Baril DT, Oakley GJ, Makaroun MS, Chaer RA. Vascular wall invasion in neurofibromatosis-induced aortic rupture. Vasc Endovascular Surg 44: 52-55, 2010.

9. Borislow DS, Floyd WL, Sane DC. Primary aortic sarcoma mimicking aortic dissection. Am J Cardiol 64: 549-551, 1989.

10. Naughton PA, Wandling M, Phade S, Garcia-Toca M, Carr JC, Rodriguez HE. Intimal angiosarcoma causing abdominal aortic rupture. J Vasc Surg 53: 818-821, 2011.

The Internal Medicine is an Open Access journal distributed under the Creative Commons Attribution-NonCommercial-NoDerivatives 4.0 International License. To view the details of this license, please visit (https://creativecommons.org/licenses/ by-nc-nd/4.0/).

(C) 2021 The Japanese Society of Internal Medicine Intern Med 60: 269-274, 2021 